\title{
COMPUTER SIMULATION OF A THIN MAGNETIC FILM WITH VERTICAL ANISOTROPY
}

\author{
J.H.J. van OPHEUSDEN ${ }^{1}$ \\ Center for Theoretical Physics, University of Twente, P.O.B. 217, 7500 AE Enschede, The Netherlands
}

and

\author{
E.M.C.M. REUVEKAMP ${ }^{2}$ \\ Low Temperature Physics, University of Twente, P.O.B. 217, 7500 AE Enschede, The Netherlands
}

Received 9 January 1990

\begin{abstract}
We describe a discrete micromagnetic model for a thin magnetic layer which has been developed to perform computer simulations of the system. The magnetisation in this model is given in terms of a cubic array of inieracting microscopic spins. The dynamics of the spins is given by a time discretisation of the Landau-Lifshitz-Gilbert equations of motion. The array is continued periodically in the $x$ - and $y$-direction in order to reduce boundary effects, and is finite in the $z$-direction. The mutual interactions that are incorporated are exchange and dipole interaction, and the crystal lattice interaction is modeled by a roughly vertical uniaxial anisotropy term. The strengths of the different interactions are scaled so as to conform to values for $\mathrm{CoCr}$, fitted to experimental results within the context of continuum models. For this setup we have determined full hysteresis curves and compared with experimental results of these films.
\end{abstract}

\section{Introduction}

In this paper we will discuss simulations of a magnetic medium consisting of a thin layer with vertical anisotropy. Such a system is relevant for the description of $\mathrm{CoCr}$ films which are made of small crystalline rods, perpendicular to the film normal. Both the crystal anisotropy and the rod geometry give rise to a vertical easy axis. The applicability of magnetic recording media with vertical anisotropy rests on the fact that they can in principle have a very large information density. For several materials also magneto-optic recording is possible. The problem that arises is the large demagnetising field at the surface. With our simu-

1 Present address: Dept. of Chemistry, University of Utrecht, Padualaan 8, $3584 \mathrm{CH}$ Utrecht, The Netherlands.

2 Author to whom requests for reprints should be sent. lation we want to obtain more explicit information about these suriace effects.

In magnetic systems as we are considering domain structures appear at a scale which is several orders of magnitude higher than atomic scales. In fact there are four levels of description. The lowest is the atomic level. The second is a level on which the quantum fluctuations are averaged out, the classical micromagnetic level. A third level describes the organisation of the system in domains, like stripes and bubbles. This is what we call the mesoscopic level. This level is with current techniques to some extent accessible to direct measurements. Finally there is the macroscopic level. where the actual application as a recording medium takes place.

In these computer simulations micromagnetic phenomena as well as macroscopic observables can be determined. By direct comparison with 
experimental results one can obtain insight in the the validity of the model which has been used. If necessary model parameters may be fitted to match the observed behaviour. On the other hand the detailed microscopic information may lead to new kinds of experiments. One advantage of numerical modelling for instance is that it is very easy to obtain information about different layers in the thin film, whereas experimentally only the upper few layers are accessible.

Computer simulation is a way of manufacturing an idealised model system which is open to observations (or rather calculations if one prefers) with in principle arbitrary detail and precision. In practice of course both are restricted because of CPU-time and memory requirements. In our model we consider discrete classical magnetic spins, placed at the vertices of a cubic lattice, and study their dynamical behaviour. It appears that even relatively small model systems show complicated behaviour. In a separate paper we analyse in detail systems from one single spin up to a $5 \times 5 \times 1$ array of spins, so we can understand the working of the model at the lowest scales [1]. Here we use arrays of $10 \times 10 \times 1$ and higher in order to be able to compare with experimental results. The practical limit for our computer system, an Apollo DN-4000 workstation with numeric coprocessor, is of the order of 1000 spins, mainly for reasons of computational speed. On faster machines, especially vector- or parallel computers, much larger systems can be considered. We think we first need more understanding of the smaller systems, especially the influence of the effective values of the parameters on the model behaviour, before it seems sensible to go to these larger machines. In this first paper we will describe some details of the program, and perform a parameter study of the system under consideration. We calculate hysteresis loops for various values of the model parameters. Next we describe some numerical experiments of a model with parameters suitable for a thin $\mathrm{CoCr}$ film, and investigate the effect of the film thickness, that is the introduction of several layers in the simulation.

Similar work on micromagnetic simulations has been done by Mansuripur [2] and Zhu and Bertram $[3,4]$. The first has simulated amurphous RE-TM thin films for magneto-optic recording, the second have been working on films with uniaxial anisotropic crystallites, both randomly [3] and perpendicularly oriented [4] as in $\mathrm{CoCr}$ films. In all these cases the Landau-Lifshitz-Gilbert equations of motion describe the dynamics of the magnetisation, using a finite damping $[2,3]$ of the order unity or an infinite damping [4]. These simulations basically are 2-dimensional. At the moment only studies of ferromagnetic fine particles are known to be 3-dimensional. For particle simulations a distinction can be made between those using LLG dynamics and those using an iteration method for energy minimalisation. Victoria [5] and Uesaka et al. [6,7], using LLG equations, focus on barium ferrite particles. Schabes and Bertram [8,9], simulating ferromagnetic cubes and elongated $\gamma-\mathrm{Fe}_{2} \mathrm{O}_{3}$ particles, and $\mathrm{Yan}$ and Della Torre [10,11], modeling also $\gamma-\mathrm{Fe}_{2} \mathrm{O}_{3}$ and $\mathrm{BaFe}$ particles, belong to the second category. Finite element calculations on (prolate) spherical small particles are performed by Fredkin and Koehler $[12,13]$.

\section{Model}

As a model for micromagnetism we use a discretised version of the Landau-Lifshitz-Gilbert (LLG) equations of motion, that describe the dynamics of the system on the basis of the magnetic energy. In continuum models the magnetisation of the sample is described in terms of a classical vector field $m(r, t)$. In our model we discretise this field in the sense that we consider a cubic array of classical magnetic spins, with fixed moment $|m|$, but with arbitrary orientation. In the $x$ - and $y$-direction periodical boundary conditions are applied, in the $z$-direction the lattice is finite.

The inagnetic energy $E\left(m_{1}, \ldots, m_{N}, B_{\text {ext }}\right)$ of the spins contains three essentially different terms: interaction with the external field $B_{\text {ext }}$, interaction with the crystal lattice and mutual interaction between the spins. The last term has two parts, an effective short range ferromagnetic exchange interaction of the form

$$
E_{\text {exchange }}=-A \sum_{\text {neighbour pairs }} m_{i} \cdot m_{j}
$$


and a long range dipole-dipole interaction term of the form

$$
\begin{aligned}
E_{\text {dipole }}= & \frac{\mu_{0}}{4 \pi} \sum_{\text {pairs }}\left[m_{i} \cdot m_{j}-3\left(m_{i} \cdot \hat{r}_{i j}\right)\right. \\
& \left.\times\left(m_{j} \cdot \hat{r}_{i j}\right)\right] / r_{i j}^{3},
\end{aligned}
$$

with $r_{i j}=r_{i}-r_{j}$ and $r_{i}$ the Euclidean positions of the spins, and $\hat{r}_{i j}$ a unit vector in the direction of $r_{i j}$. The dipole field is often called the demagnetising field. Note that all pairs interact, using the periodic boundary conditions in calculating their mutual distance. In the present simulations we have used a hard cutoff at the boundary of the image box, in a later paper we investigate the effect of correction terms for the neglect of far field dipole contributions.

Next there is a phenomenological interaction with the crystal lattice in which the atomic spins are embedded, which we have taken to be a uniaxial anisotropy field

$E_{\text {anisotropy }}=-\sum_{\text {spins }} K_{i}\left(m_{i} \cdot a_{i}\right)^{2}$

The unit vector $a_{i}$ gives the direction of the easy axis, which in most of our simulations is almost vertical, but in principal it can point in an arbitrary direction. Also the interaction strength $K_{i}$ may differ from one spin to another. Most importantly both can differ between layers in order to simulate double layer materials, but also defects and other discontinuities can be taken into account. Note that the interaction with the crystal lattice does not assume a cubic crystal, only the discretisation of the spin lattice is cubic.

Finally the interaction with the external field $B_{\text {ext }}$ is given simply by

$E_{\text {external }}=-\sum_{\text {spins }} m_{i} \cdot B_{\text {ext }}$.

The external field is constant over all spins, but it may vary with time in order to investigate the dynamical behaviour of the system under changes in the field, especially in hysteresis loops.

The dynamics of the spins is given by the LLG equations, that apart from the Larmor precession of the magnet include a phenomenological damp- ing term describing the relaxation into the direction of the local magnetic field. The equations of motion for a single spin are of the form [14]

$\mathrm{d} m / \mathrm{d} t=-\gamma m \times B_{10 c}-\alpha m \times\left(m \times B_{10 c}\right)$,

where the quantity $m$ is the magnetisation vector of the individual spins. The local magnetic field of spin $m_{i}$ is defined as

$B_{\mathrm{loc}}=-\partial E / \partial m_{i}=B_{\mathrm{ani}}+B_{\mathrm{exc}}+B_{\mathrm{dip}}+B_{\mathrm{ext}}$,

where the first term is determined by the direction of the spin itself with respect to the anisotropy axis, while the second and the third term are given by the values of the neighbouring spins and the distant spins, respectively.

\section{Computer program}

The equations of motion as given by (5) are integrated numerically. We have applied an algorithm which makes use of the fact that for a constant local field the LLG equation can be solved analytically, and a closed form solution of $m(t)$ can be found in terms of $m(0), B_{\text {loc }}$ and the model parameters $\alpha$ and $\gamma$. A description of this solution is given in appendix B. In our si.nulation we take the local field, which is given as a function of the $m_{i}$, constant for small time intervals $\Delta t$, then calculate the new values of $m_{i}$ and repeat our calculation. The external field is also considered constant during the step, but can be changed between steps in order to study hysteresis effects. The effective damping parameter $\alpha$ can have any value within this algorithm. Not only the phenomenological value can be used, but also the limit $\alpha \rightarrow \infty$ is valid. Very large values of $\alpha$ can be considered when not the dynamics, but only the effect of a very slow change in the external field on the stationary state is of interest. In the latter case we will speak of the quasi-static or adiabatic approximation.

The programs were run on an Apollo DN-4000 workstation with numeric coprocessor, operating at a speed of approximately $0.3^{\circ} \mathrm{Mflops}$. In order to be able to run the program on such a small system we had to perform some further optimiza- 
tion with respect to the integration mechanism. Most of the computing time is spent on evaluating the dipole field. Note that there is an important distiction between the dipole field of our model with discrete magnets and the dipole field of models with a continuous magnetisation. In the latter case the restriction that $\operatorname{div} m=0$ within the sample leads to the result that only effective magnetic charges at the interface produce the demagnetising field. For the discrete model there is no analogous concept that gives the same reduction, so we have to calculate the dipole fields of each individual spin at the position of all the other spins. The same disadvantage, by the way, applies for the cellular models which are used elsewhere.

The optimisation step we have introduced is to consider the dipole field of each individual spin at the position of the other spins,

$$
\begin{aligned}
B_{\text {dipole }}\left(m_{i}, r_{j}\right)= & \left(-\mu_{0} / 4 \pi\right) \\
& \times\left[m_{i}-3\left(m_{i} \cdot \hat{r}_{i j}\right) r_{i j}\right] / r_{i j}^{3},
\end{aligned}
$$

and only recalculate this field when' $m_{i}$ has changed considerably, within limits to be specified at execution. The rational is that for instance in particulate systems each individual particle will have a fairly constant magnetisation until the local field reaches a certain level, the reversal field strength, upon which a spin flip occurs. When the anisotropy field in our model is dominant in the sense that each spin has two equivalent minima, up or down, one may expect similar behaviour. As it of ten happens in the simulations at each step most spins are rather fixed, and few spins are moving quite rapidly. In these cases our trick leads to a substantial reduction of the computing time. The method fails if there is coherent behaviour of large numbers of spins. In that case a small change in the individual spins is enhanced by the collective motion, and the approximation breaks down. Indeed for small systems we have ohserved in some instances, even for a fixed external field, very distinct oscillatory behaviour of the magnetisation, due to neglect of a coherent change in the motion of the spins. The same optimisation can of course be used on larger machines, that is if it does not interfere with parallel computing methods.

\section{Computer experiments}

Because the scale of the model can be chosen at will, at least in principle one has the option to use the model on various levels of detail. If one takes $\Delta x$ of the order of a domain size the granular structure of the sampl $\mathrm{is}$ dominant, and the simulation would apply best for particulate systems. On the other hand one could choose $\Delta x$ to be of the order of the distance between spins in the real sample, and investigate in detail the structure of a domain wall. In our present simulations, as we have indicated in the introduction, we have chosen an intermediate level, with $\Delta x$ of the order of the width of a domain wall. At this level the LLGequations of motion are appropriate for the description of the dynamics of the magnetisation of the sample.

We have chosen an actual lattice spacing of $\Delta x=16 \mathrm{~nm}$. In the calculations all variables are dimensionless. In the program we use unit lattice constant and unit spins. The actual magnetic moment associated with a discrete spin becomes $m=$ $m_{\mathrm{s}} \Delta x^{3}$, with $m_{\mathrm{s}}=4 \times 10^{5} \mathrm{~A} / \mathrm{m}$ the saturation magnetisation of $\mathrm{CoCr}$. The dipole field is also dimensionless, so there is a prefactor $\mu_{0} m_{s} / 4 \pi=$ $1 / d^{3}$ with $d \Delta x$ the effective neighbour distance. The parameter $d$ scales $m_{\mathrm{s}}$ rather then the length, and $\Delta x$ does not appear in the dipole term. The same is true for the anisotropy term, because both $K$ and $m_{\mathrm{s}}$ are densities, so any factors $\Delta x$ disappear in the prefactor $K_{\text {cal }}=K_{\exp } / m_{\mathrm{s}}$. For $\mathrm{CoCr}$ we take $K_{\text {exp }}=1.6 \times 10^{5} \mathrm{~J} / \mathrm{m}^{3}$. The exchange term does depend on $\Delta x$, because $A \sim 1 / \Delta x$ we find that the prefactor becomes $A_{\text {cal }}=A_{\text {exp }} / m_{\mathrm{s}} \Delta x^{2}$. For CoCr we have taken $A_{\exp }=10^{-11} \mathrm{~J} / \mathrm{m}$.

The first experiments that we will describe, are performed upon a single layer of spins. For this system we have varied several model parameters; the damping, the system size, the exchange and dipole coupling and the anisotropy strength. The values of the physical parameters were such as to approximate those of a $\mathrm{CoCr}$ sample. The results of this parameter study are described in the next section.

In all cases we have started from a configuration with the spins in the positive $z$-direction, and with the external field at a value in which the 
system is near saturation. Then the field was lowered gradually to the opposite value, while the dynamics of the system is integrated in order to have the sample magnetisation follow adiabatically. For the full curve the external field should be brought back again to the positive value, but because of the inherent symmetry of the model this would not add much information. For some trial samples we have checked that indeed this is the case. Hence in further calculations we skipped this part, and simply plotted both the normal and the reversed path in our graphs, for convenience only.

In the second experiment we have fixed the parameter values to those of $\mathrm{CoCr}$. Note, however, that because these values were measured within the context of continuum type models there still may be a slight difference with the effective values we should use in our discrete model. For a single layer of spins we have calculated several inner loops in a hysteresis curve.
Finally, we have investigated the effect of the introduction of a second and a third layer to the model system, both for the global hysteresis curve, as for the actual detailed configuration as a function of the external field.

\section{Results}

The first parameter study we have' performed concerned the value of the friction that should be chosen in order to have the system perform adiabatic motion. We have investigated several values of the friction from $\alpha=0.1$ to $\alpha=300$. Because $\alpha$ as we use it, is not really a physical parameter we have not included the detailed graphs. The result is that for $\alpha>10$ there is hardly any difference at the level of the hysteresis curve, and we take the adiabatic approximation to be valid. For smaller values there are distinct effects caused by the large relaxation time of a single spin as compared to the

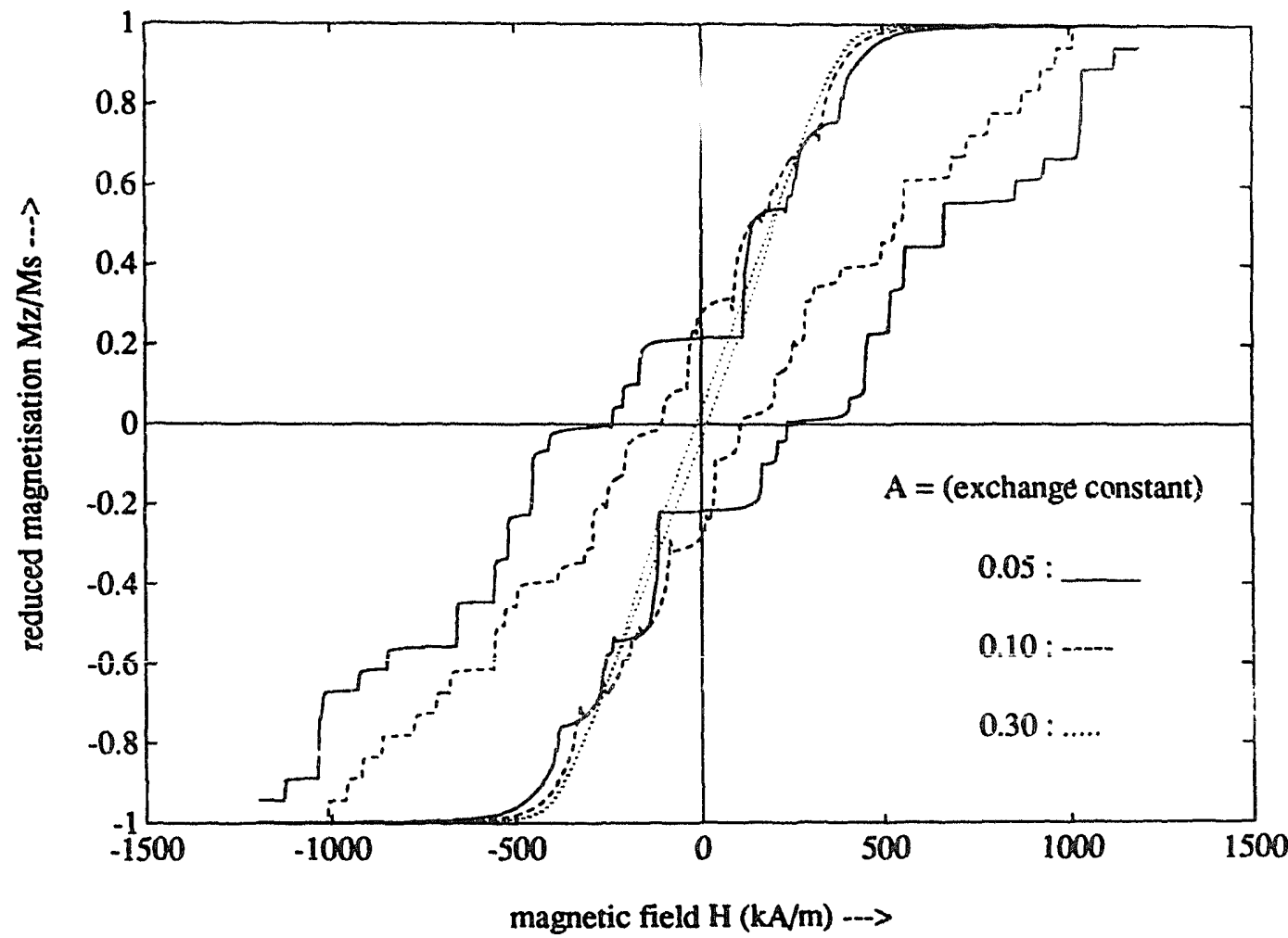

Fig. 1. Hysteresis curves for a $6 \times 6 \times 1$ sample with vertical anisotropy at different exchange constants. Other parameter were the anisotropy $K=0.5$, and dipole parameter $d=1.9$. For an explanation of the various parameters see text. 


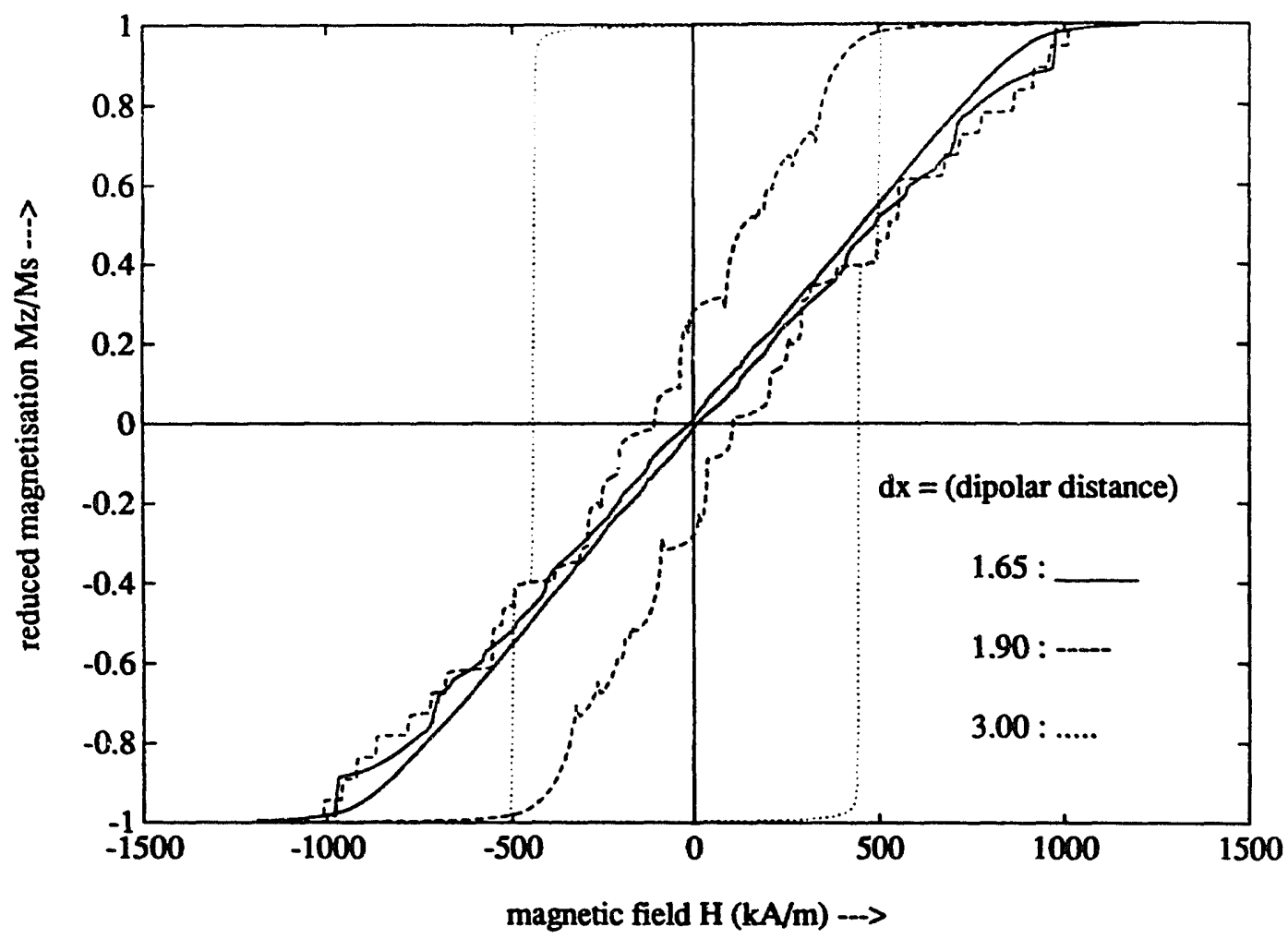

Fig. 2. Hysteresis curves for various values of the dipole parameter. The dipole strength decreases with increasing $d$. The exchange parameter is $\boldsymbol{A}=0.1$, other parameter values as in the previous graph.

rate at which the external field is changed. One main effect which we have observed is that when one spin flips, the change in its dipole field will slightly redirect its neighbours in the antiparallel direction, and so forth. Hence small waves move out from the flipped spin, and these waves have to relax before the new equilibrium state is obtained. For the friction valces as we have used this behaviour is mostly repressed, or remains restricted to one or two integration steps only. In the hysteresis curves this appears as an overshoot effect, where the magnetisation drops below its equilibrium value, and the curve seems to rise with decreasing external field, at least for small systems. See for instance the $A=0.10$ curve in the next experiment. For larger system sizes the effect is averaged out, or disappears in the overall noise.

The following three series of parameter studies were performed upon a $6 \times 6 \times 1$ array of spins, a single layer continued periodically in the $x$ - and $y$-direction. For this system we have taken three different values of the exchange coupling strength, parameter values $A=0.05,0.10$ and 0.30 , respectively (fi $i_{\text {s. }} 1$ ). The other parameters were the dipole strength parameter $d=1.90$, the anisotropy strength $K=0.5$ and the friction coefficient $\alpha=$ 30 . Note that $A$ is the only parameter that changes with the scale, for low $A$ the individual spins stand for objects the size of a domain, while for large $A$ the spins act as micromagnets and only part of a domain wall is modelled.

The value of $A=0.10$ is that of $\mathrm{CoCr}$. For that value we observe that the system takes on a structure where the spins are directed along a single vortex-antivortex pair. At the vortex the spins remain in the up position, and away from it the in-plane component increases. The spin flips occur far away from the vortex, which acts as a pinning centre. After a spin has flipped the vortex structure relaxes, but remains intact, because of the symmetry of the anisotropy term. The in-plane configuration has a low dipole energy, the vertical position a low anisotropy energy. The exchange between these terms by a spin flipping over can 


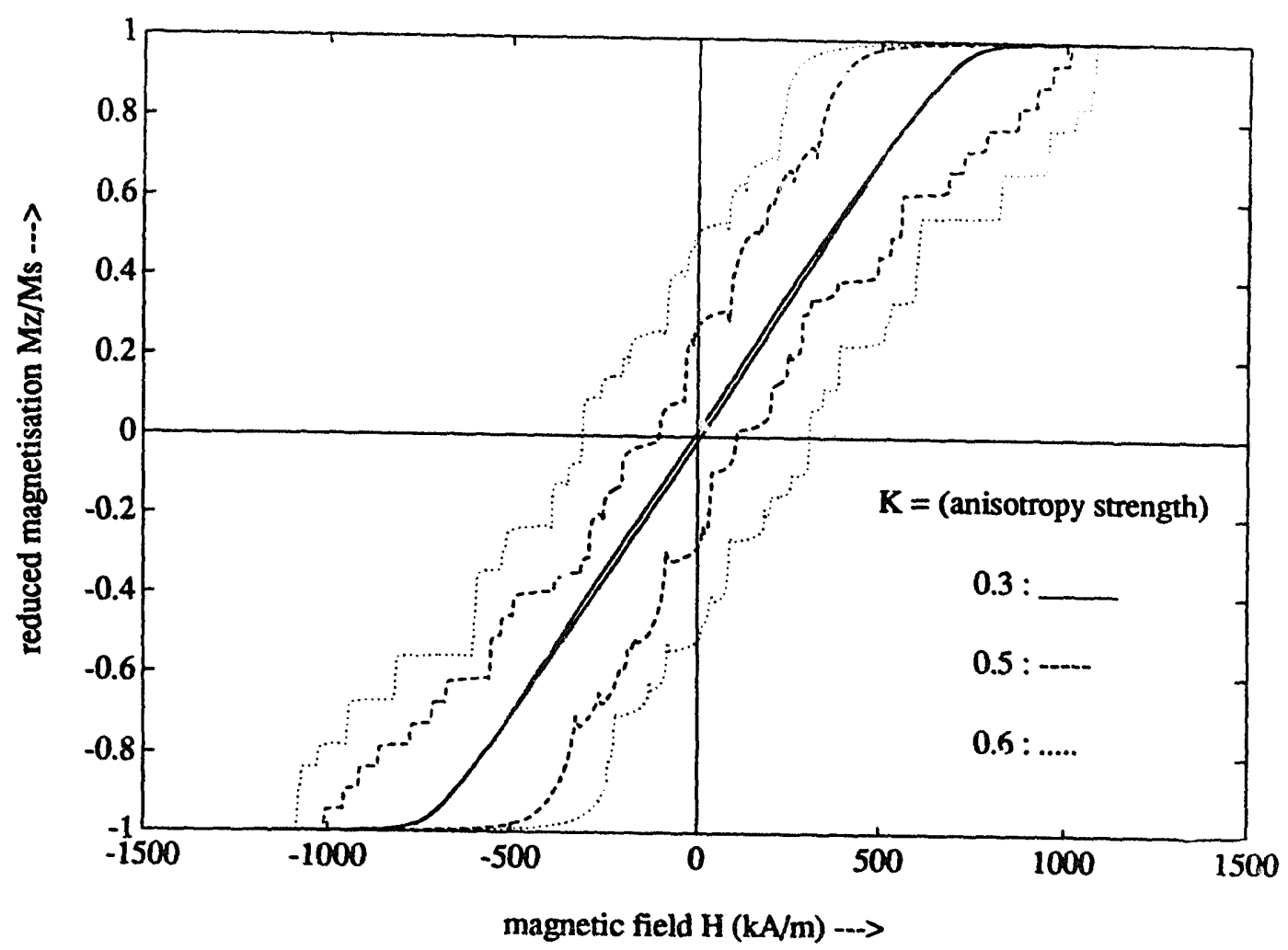

Fig. 3. Hysteresis curves for different values of the anisotropy. Other values are $K=0.5$ and $d=1.9$.

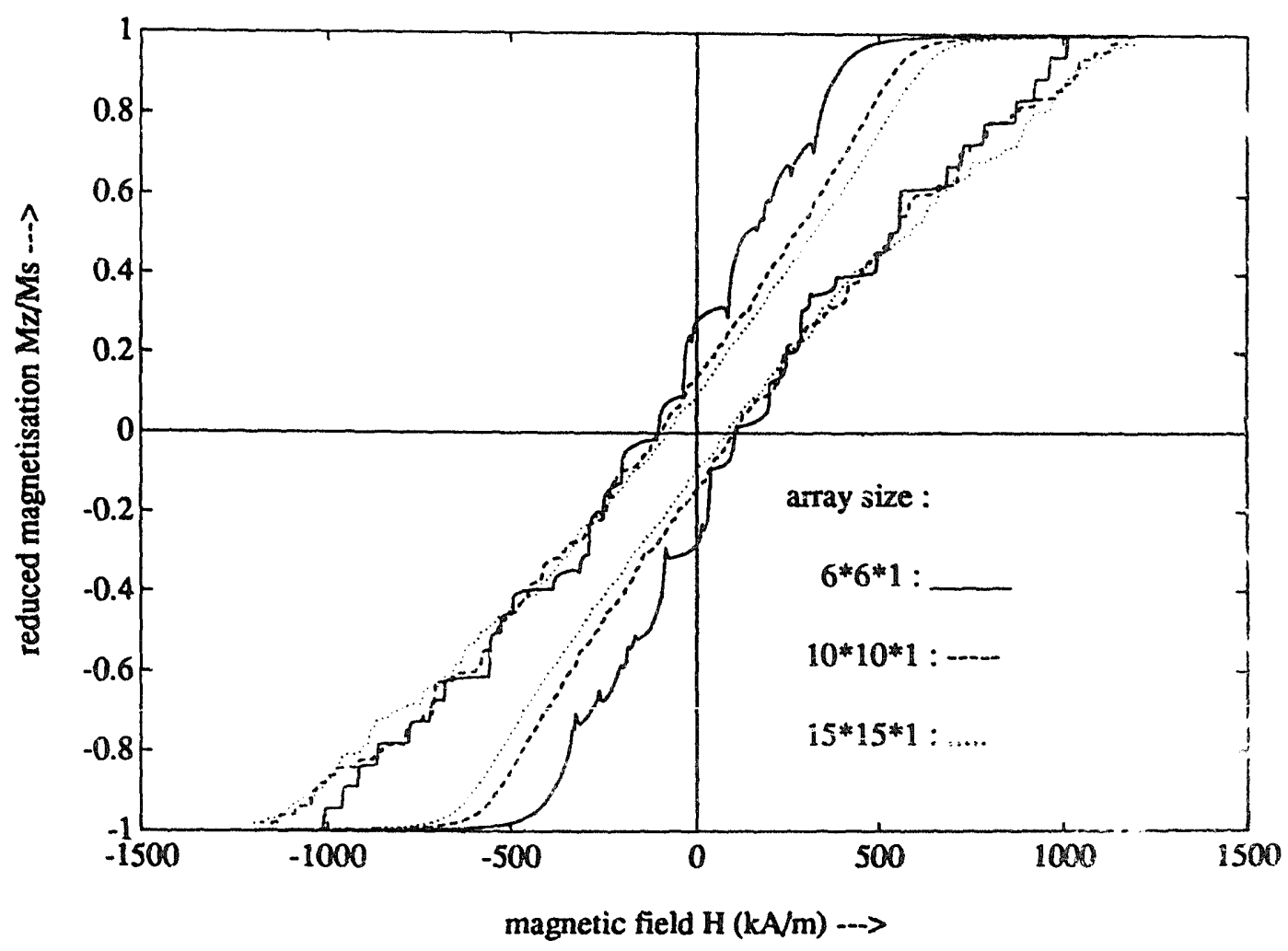

Fig. 4. Hysteresis curves for various model system sizes. The difference is an effect of scale, becarse size effects are reduced by imposing periodical boundary conditicns. Parameters $A=0.1, K=0.5$ and $d=1.9$ were chosen to compare with CoCr. 


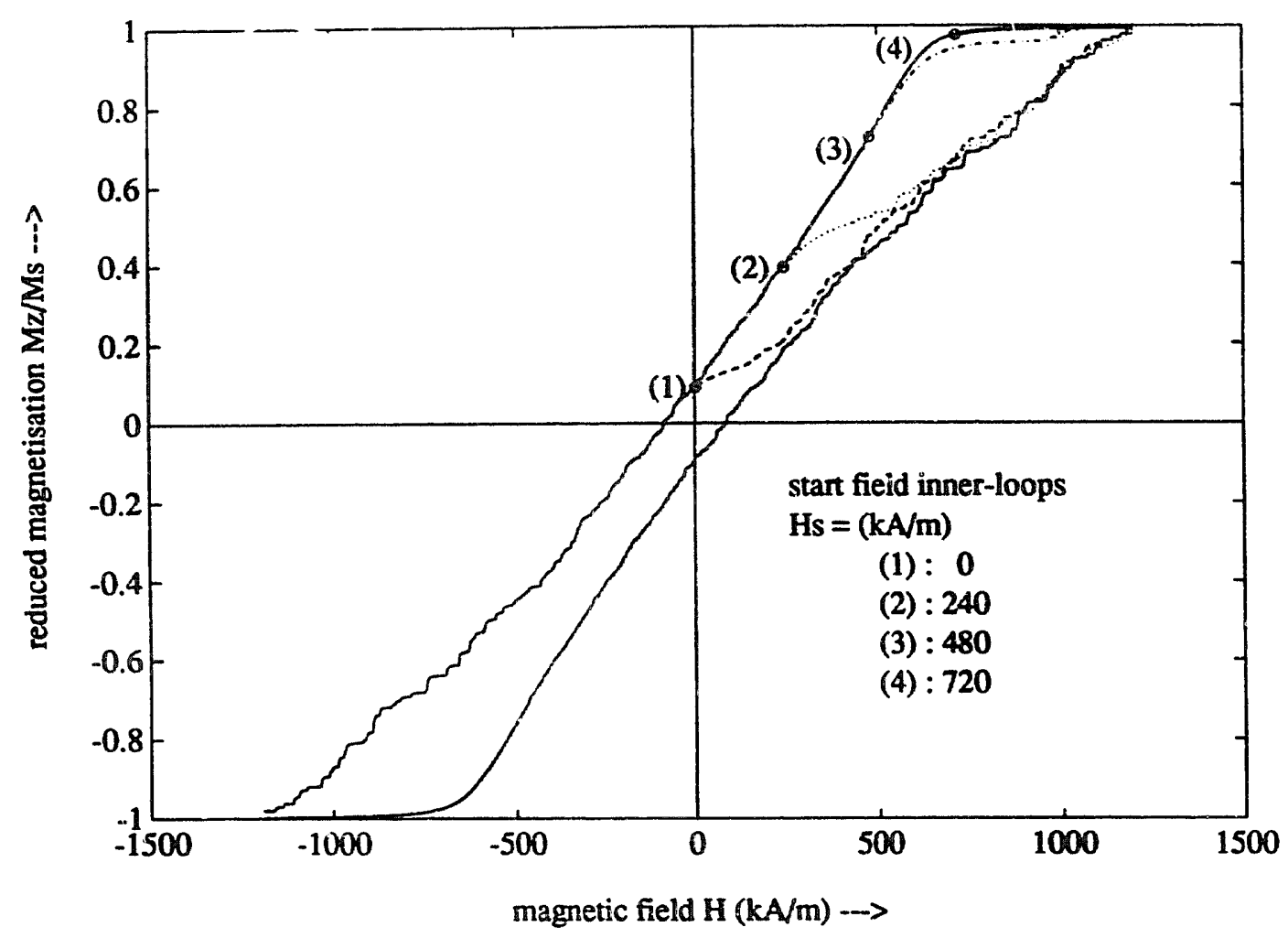

Fig. 5. Inner loops for the hysteresis curve of a $15 \times 15 \times 1$ array. Parameters as in $\mathrm{CoCr}$.

also be regarded as the formation of a domain wall, followed by a relaxation of the vortex structure.

For lower $A=0.05$ the finite system size become apparent, and individual spin flips can be observed. The vortex structure is still present, but much less pronounced. Sometimes several spins flip at the same time, but these are not necesarily neighbours on the lattice, in fact in most cases they are not. Actually an alternation of up and down spins in an checkerboard pattern appears for states with a low total magnetisation. The hysteresis curve is rather broad because of the stability of the patterns, and the external field we have applied apparently is not strong enough to pull the system to its negative saturation value

For a high value of $A=0.3$ there is a uniform rotatiuni; the sample. 'This can be seen as a simple sca effect. A higher $A$ implies a smaller scale, so we should expect this more coherent behaviour. The curve shows only a negligibly small hysteresis effect.
The next series (fig. 2) concerns the value of $d$, for which we have taken $d=1.6,1.9$ and 3.0. Note that the dipole strength is inversely proportional with the third power of $d$, so the variation is rather large. The medium value is already discussed above. For the low value $d=1.6$ (high dipole coupling), the vortex structure is very pronounced, and the spins rotate to an in-plane configuration, even for relatively large external field. The hysteresis effect is small and the system is easily brought out of its saturation state. For the high value $d=3.0$, the dipole coupling is very small, and the system is dominated by the exchange and anisotropy effect. The result is that the saturation state, which is stable under small periurbations for both latter interactions, becomes unstable only for large negative external fieitl. When that happens the reversal occurs for all spins simultaneously, because the anisotropy is almost identical. The exchange enhances the effect. The hysteresis curve is broad, and almost like that for a single spin. 


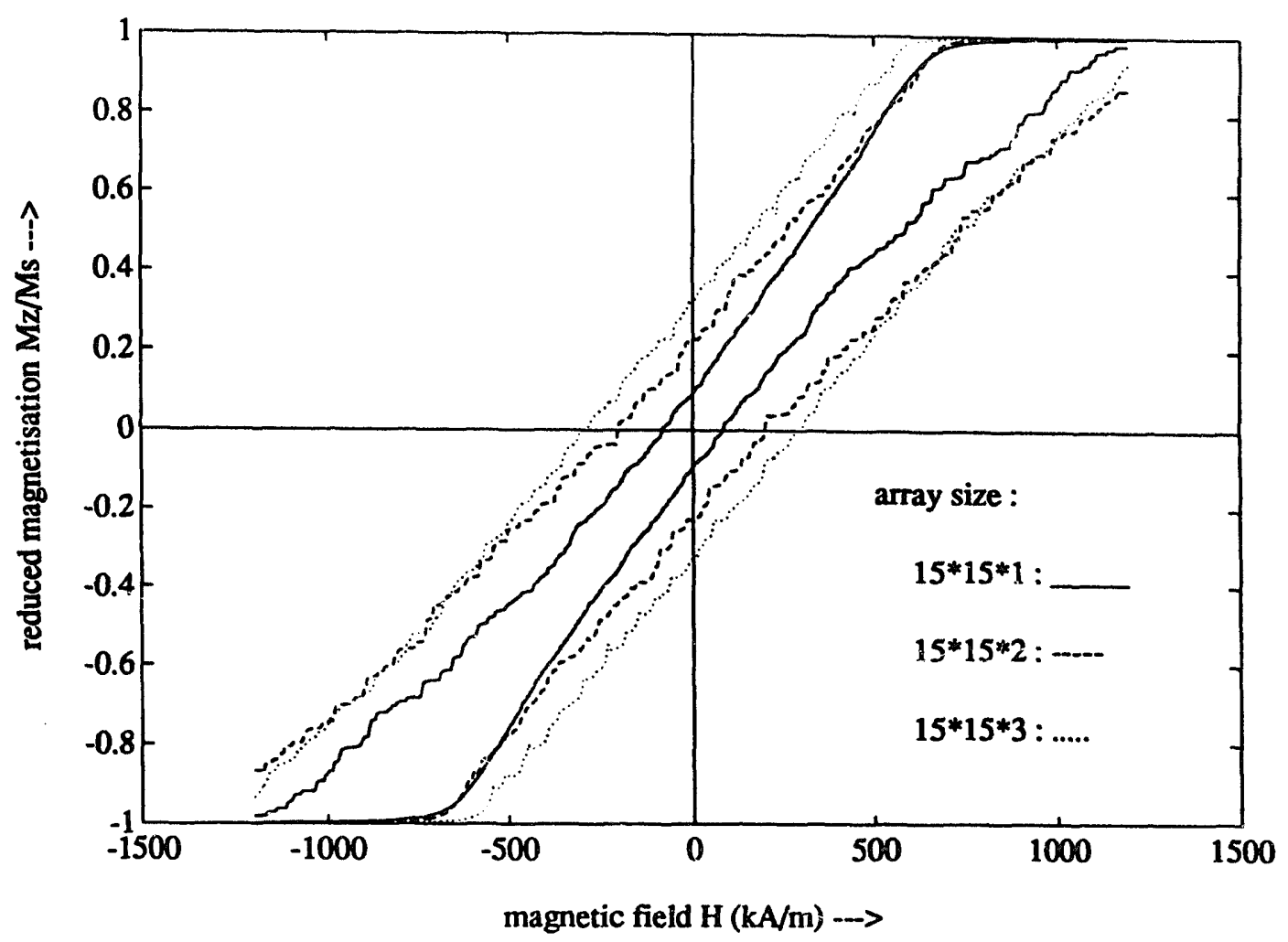

Fig. 6. Hysteresis curves for samples of different number of layers, parameters as in CoCr.

The next parameter (fig. 3) we have varied is the anisotropy strength for which we have taken $K=0.3,0.5$ and 0.6 . The middle value again has been discussed above. For the lower value the vortex structure does form, but the pair is asymmetrical, one vortex is much stronger than the other (anti-)vortex. The result is that a stripe domain structure appears, with mostly in-plane magnetisation. Because the anisotropy is small the saturated state is easily destabilised, and also the hysteresis effect of this curve is very small. For the higher value of $K=0.6$ the behaviour is rather like that for $K=0.5$, only things appear at somewhat lower values of the external field. We admit that the difioranos between the values is rather small too, but :or still higher $K$ no new effects were found.

In the next series (fig. 4) we have changed our system size, which is a parameter of the model system, rather than a physical value of our sample. The main effect that can be observed is an overall smoothing of the curve, which is caused iy the fact that more vortex pairs are present in the system. A still larger system of $30 \times 30 \times 1$ is discussed in an earlier paper of us [15]. For the largest systern in our experiment we have calculated four inner loops of the hysteresis curve, starting at different values of the external field (fig. 5). One observes that for the highest value the process is reversible, because no spin flips have occurred yet. For the first value below that one there have been some spin flips, but they are reversed before the external field reaches its saturation value. The irreversible effect is small. For the lowest two values we have investigated the curve crosses completely to the reverse branch. This experiment shows clearly that our model system has various stable states, with different magnetisation, for the same value of the external field.

The last series of experimenis to be discussed in this paper has been performed upon a $15 \times 15$ system of two and three layers. The results are comprised in fig. 6 . One may observe that contrary to expectation the discrete steps in the curve become more pronounced, rather than less. The 

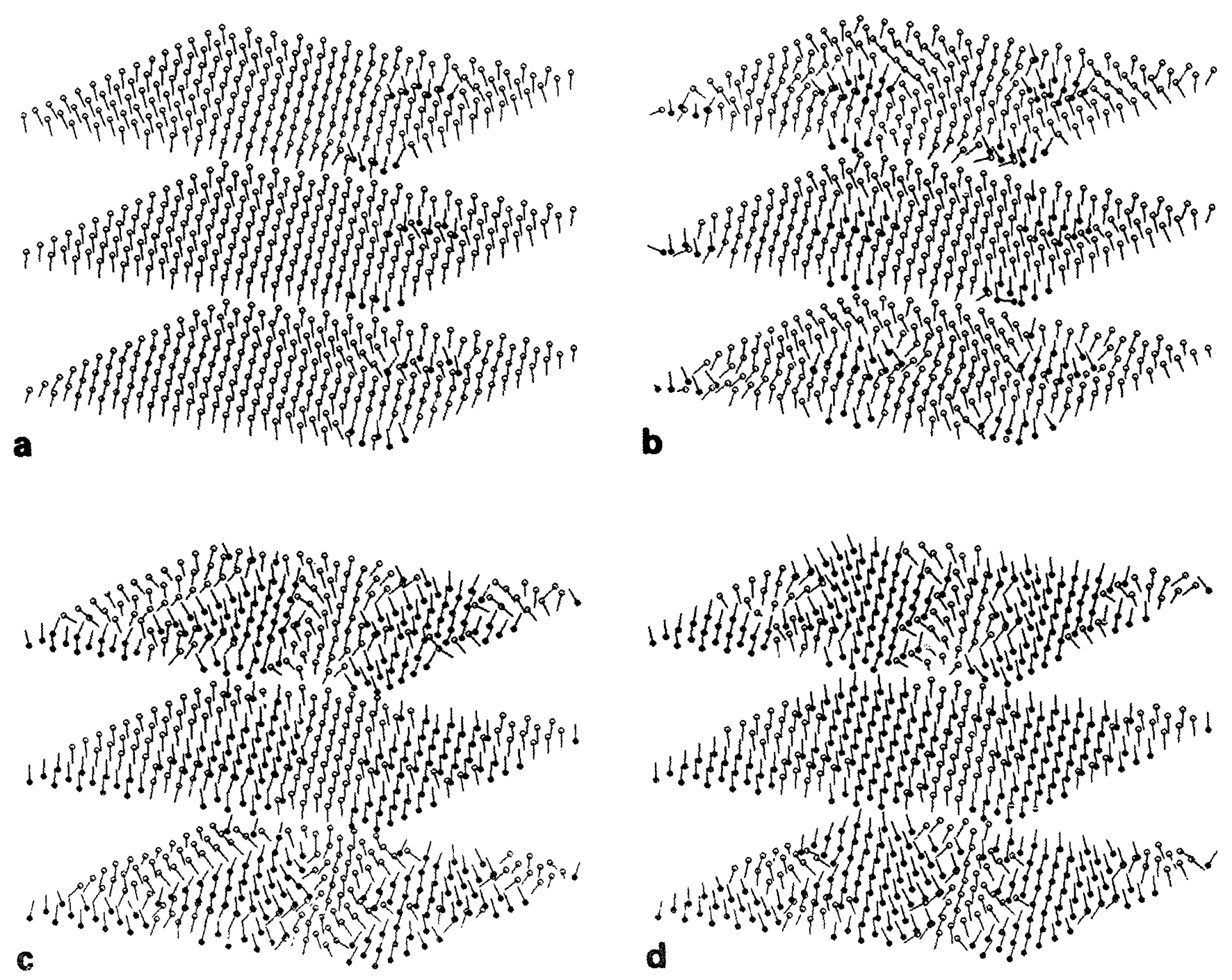

Fig. 7. Configurations of the $15 \times 15 \times 3$ system along the upper branch of the hysteresis curve at field values $H=500$ (a), 250 (b), -250 (c) and $-500 \mathrm{kA} / \mathrm{m}$ (d). Spins are drawn with their centres fixed to the lattice. Upward pointing spins are indicated by open circles, downward pointing spins by filled circles.

reason is that the vortex structure in this system becomes larger, and hence the discreteness is more apparent. Apart from being larger the vortices also have a structure in the $z$-direction. A vortex in the upper layer is an anti-vortex in the lower one, that is the orientation is a function of the perpendicular direction. In the three layer sample the effect was that the spins in the middle layer were directed almost completely along the z-axis. A series of plots of this system for four different values along the upper branch of the hysteresis curve (figs. $7 a-d$ ) shows this effect rather clearly.

\section{Conclusions}

The vortex mechanism we have advocated in the previous section is reminiscent of the Curling mechanism, which was used in eariier numerical studies performed at our institute [16]. One difference is that the vortex mechanism is not fully symmetric. There are discrete nucleation points though that may be a lattice artefact. Also th vortex may move in the lateral direction, and relaxes after nucleation has taken place. Mo importantly maybe is tha: for systems with $\mathrm{mc}$ 


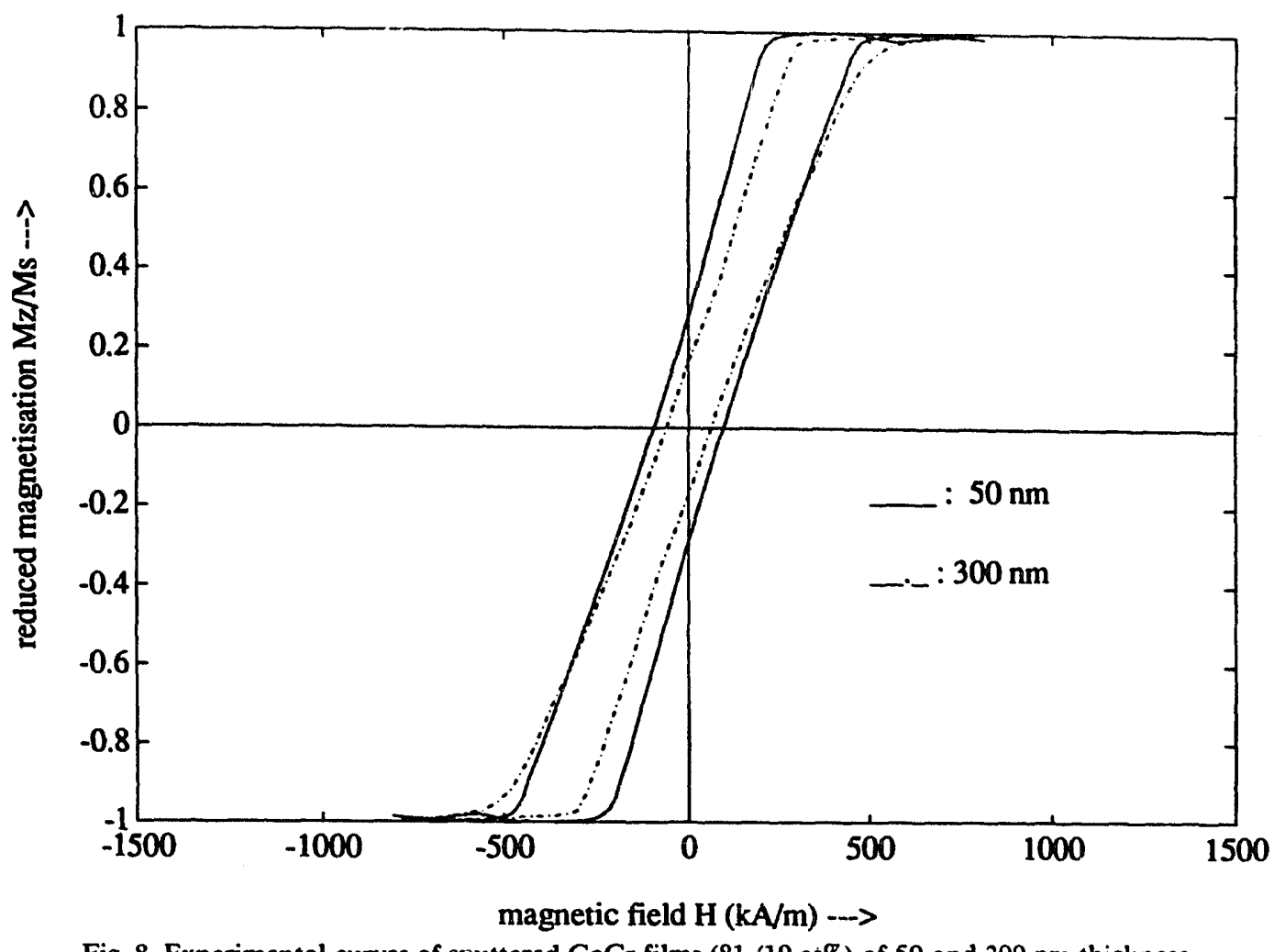

Fig. 8. Experimental curves of sputtered $\mathrm{CoCr}$ films (81/19 at\%) of 50 and $300 \mathrm{~nm}$ thickness.

layers the orientation of the vortex differs between layers, and the vortex line is not always perpendicular to the film. We have shown that this mechanism always occurs, but other effects may dominate over it for very high or low system parameters.

If we compare our curves in fig. 6 with those of actual expermental results on $\mathrm{CoCr}$ films of different thickness (fig. 8) we can conclude that our model also gives a fairly good qualitative description of what could happen in the real world. The experimental curves are similar to our numerical results, the quantitative difference may be relaxed by fitting of the remaining model parameters. In fact raising only the $d$-parameter to a value of about 2.2 would give rather good agreement. As already stated one could have expected such an effect when transforming experimental values that are defined within a continuum context to numerical parameter values in a discrete model.

What also can be seen in the experimental curve is that the thin film is more like our triple layer sampler, while the thicker one looks more like our single layer calculation. One reason could be that for a single layer the particle-like behaviour is somewhat dominant, like one may expect to be the case for a thicker real sample. The multilayer calculation would be a better approximation for the continuum system, and hence give a better comparison with the thin films. Of course many other effects may cause the difference in behaviour between the experimental samples, and much further study is necessary to make the subject clearer.

\section{Appendix A. Equivalence of damping terms}

There are ir general two ways one uses to introduce damping in a system of magnetic spins. To the undamped equation of motion in a local field $B=-\partial E_{\mathrm{mag}} / \partial m$

$$
\mathrm{d} m / \mathrm{d} t=-\gamma m \times B
$$

one adds a term which is linear in $\mathrm{d} m / \mathrm{d} t$ and 
perpendicular to $m$ in order to conserve the vector length. The Landau-Lifshitz damping is

$\mathrm{d} m / \mathrm{d} t=-\gamma m \times B-\alpha m \times(m \times B)$,

and the Gilbert damping is of the form

$\mathrm{d} m / \mathrm{d} t=-\gamma m \times B+\alpha / \gamma(m \times \mathrm{d} m / \mathrm{d} t)$.

In fact there is no real distinction between the two forms, apart from the fact that the constants $\alpha$ and $\gamma$ are defined somewhat differently, but after all these are only fitting parameters as far as the calculation is concerned. If the constants are interpreted in terms of physical variables, however, the distinction is very real [17]. That the two equations are fully equivalent can be shown by taking eq. (A.2b), bringing the second term of the right hand side to the left and operating on both ends with the operator $"+\alpha m \times)$. The result is that the right hand side has the form of eq. (A.2a), while the left hand side is

$$
\begin{gathered}
\mathrm{d} m / \mathrm{d} t+\alpha^{2} / \gamma(m \times(m \times \mathrm{d} m / \mathrm{d} t))=\left(1+m^{2} \alpha^{2} / \gamma\right) \\
\mathrm{d} m / \mathrm{d} t-\alpha^{2} / \gamma(m \cdot \mathrm{d} m / \mathrm{d} t) m .
\end{gathered}
$$

Here we have used a standard vector relation to expand the double wedge product. Because $\mathrm{d} m / \mathrm{d} t$ is perpendicular to $m$ the second term becomes zero and the equivalence is proven. Any other form one would like to propose will also be equivalent for the simple reason the space normal to $m$ is only two dimensional, and any two basic vectors will always be a linear combination of the ones used above.

Appendix B. Solution of the linear equation of motion

The equation of motion we want to solve is

$\mathrm{d} m / \mathrm{d} t=-\gamma m \times B-\alpha m \times(m \times B)$.

For simplicity we take $B$ along the $z$-axis, and constant during a small time interval $\Delta t$ ( $B=$ $(0,0, b))$. The components of the unit magnetic spin are denoted by $m=(x, y, z)$. In Euclidean coordinates eq. (B.1) takes the form

$$
\begin{aligned}
& \mathrm{d} x / \mathrm{d} t=-\gamma b y-\alpha^{2} b x z, \\
& \mathrm{~d} y / \mathrm{d} t=\gamma b x-\alpha b y z, \\
& \mathrm{~d} z / \mathrm{d} t=\alpha b\left(1-z^{2}\right)
\end{aligned}
$$

The solution of eq. (B.4) is

$z(t)=\tanh \left(\zeta_{0}+\alpha b t\right)$,

with implicitly $z_{0}=\tanh \left(\zeta_{0}\right)$. The other two components become

$$
\begin{aligned}
x(t)= & \left(x_{0} \cos \omega t+y_{0} \sin \omega t\right) \\
& \times \cosh \left(\zeta_{0}\right) / \cosh \left(\zeta_{0}+\alpha b t\right), \\
y(t)= & \left(y_{0} \cos \omega t-x_{0} \sin \omega t\right) \\
& \times \cosh \left(\zeta_{0}\right) / \cosh \left(\zeta_{0}+\alpha b t\right),
\end{aligned}
$$

with $\omega=\gamma b$. Note that the damping does not influence the precession frequency around the magnetic field vector. This becomes clear when writing the equations in spherical coordinates, where the equation for the azimuthal angle $\varphi$ does not include a damping term.

This lcoks like a very intricate procedure, but it has no important effect on the computational speed as this has to be done only once for each spin at every step. The dipole field remains the dominant factor. In fact one does not even in practical computations use the special functions as tanh and cosh, which are introduced here for mathematical simplicity only. The largest contribution to the computer time for this algoritm actually does not come from these functions, but rather from the sine and cosine functions used to rotate $B_{\text {loc }}$ to the $z$-axis and back.

\section{References}

[1] J.H.J. van Opheusden, Stability of configurations in a discrete micromagnetic model, in preparation.

[2] M. Mansuripur, J. Appl. Phys. 63 (1988) 3831.

[3] J.-G. Zhu and H.N. Bertram, J. Appl. Phys. 63 (1988) 3248.

[4] J.-G. Zhu, H.N. Bertram, J. Appl. Phys. 60 (1989) 1291.

[5] R.H. Victoria, J. Appl. Phys. 63 (1988) 3423. 
[6] Y. Nakatani, Y. Uesaka and N. Hayashi, Computer simulation of magnetic reversal in fine hexaferrite particles, presented at MMM'89, DC-01.

[7] Y. Nakatani, Y. Uesaka and N. Hayashi, Dynamica! calculation of magnetic reversal in olongated particles, presented at MMM'89.

[8] M.E. Schabes and H.N. Bertram, J. Appl. Phys. 64 (1988) 1347.

[9] M.E. Schabes and H.N. Bertram, J. Appl. Phys. 64 (1988) 5832.

[10] Y.D. Yan and E. Della Torre, J. Appl. Phys. 66 (1989) 320.

[11] Y.D. Yan and E. Della Torre, IEEE Trans. on Magn. MAG-25 (1989) 3464.
[12] D.R. Fredkin and T.R. Koehler, IEEE Trans. on Magn. MAG-24 (1988) 2362.

[13] D.R. Fredkin and T.R. Koehler, IEEE Trans. on Magn. MAG-25 (1989) 3473.

[14] F.H. de Leeuw, R. van den Doel and U. Enz, Rep. Prog. Phys. 43 (1980) 689.

[15] E.M.C.M. Reuvekamp, J.M.J. van Opheusden and G.J. Gerritsma, J. Magn. Magn. Mat. 83 (1990) 59.

[16] G.T.A. Huysmans, J.C. Lodder and J. Wakui, J. Appl. Phys. 64 (1988) 2016.

[17] J.C. Mallinson, IEEE Trans. on Magn. MAG-24 (1987) 2003. 